\title{
Frustrated double ionization of argon atoms in strong laser fields
}

\author{
Seyedreza Larimian, ${ }^{1}$ Sonia Erattupuzha, ${ }^{1}$ Andrius Baltuška, ${ }^{1}$ Markus Kitzler-Zeiler, ${ }^{1}$ and Xinhua Xie (谢新华) ${ }^{1,2, *}$ \\ ${ }^{1}$ Photonics Institute, Technische Universität Wien, A-1040 Vienna, Austria, European Union \\ ${ }^{2}$ SwissFEL, Paul Scherrer Institute, 5232 Villigen PSI, Switzerland
}

(Received 28 September 2019; revised manuscript received 20 November 2019; published 7 January 2020; corrected 6 May 2020)

\begin{abstract}
We demonstrate kinematically complete measurements on frustrated double ionization of argon atoms in strong laser fields with a reaction microscope. We found that the electron trapping probability after strong-field double ionization is much higher than that after strong-field single ionization, especially in the case of high laser intensity. The retrieved electron momentum distributions of frustrated double ionization show a clear transition from the nonsequential to the sequential regime, similar to those of strong-field double ionization. The dependence of electron momentum width on the laser intensity further indicates that the second released electron has a dominant contribution to frustrated double ionization in the sequential regime.
\end{abstract}

DOI: 10.1103/PhysRevResearch.2.013021

\section{INTRODUCTION}

When an atom or a molecule is exposed to a strong laser field it may become singly or multiply ionized [1,2]. After the strong-field interaction, a fraction of the ionized electron wave packets with near-zero kinetic energies can be trapped into high-lying Rydberg states, a process also known as frustrated field ionization [3-13]. Frustrated double ionization (electron trapping after double ionization) has been experimentally studied for small molecules, including $\mathrm{H}_{2}$ and argon dimers, using Coulomb explosion imaging [6-8,14-17] and theoretically using the classical trajectory Monte Carlo method $[18,19]$. In these experiments the trapping process is identified by the kinetic energy released (KER) during the Coulomb explosion of the molecules. Since an electron trapped in high-lying Rydberg states does not fully shield the nuclear charge, the KER for a molecule with an electron in Rydberg states is higher than that for a nonexcited molecule fragmenting from the same charge state. Since this method is based on the measurement of KER from molecules undergoing Coulomb explosion, it is applicable to neither atomic targets nor molecules that do not fragment.

In this paper, using an alternative method developed in our previous work [12], we report on kinematically complete experiments of electron trapping processes during strong-field double ionization of argon atoms. Strong-field double ionization may happen sequentially, where the two electrons are removed one after another by the laser field, or nonsequentially, where the second electron is released during the recollision of the first electron with the parent ion [20-22]. We show that the trapping probability is strongly enhanced in the sequential

\footnotetext{
*xinhua.xie@tuwien.ac.at

Published by the American Physical Society under the terms of the Creative Commons Attribution 4.0 International license. Further distribution of this work must maintain attribution to the author(s) and the published article's title, journal citation, and DOI.
}

ionization regime. Based on our experimental data we explain the electron dynamics underlying these observations.

\section{EXPERIMENTAL SETUP AND METHOD}

In our experiments we employed a reaction microscope $[23,24]$ for three-body coincidence detection of two electrons and their parent ion created during the interaction of argon atoms with strong laser pulses [Fig. 1(a)]. Laser pulses linearly polarized along the spectrometer axis ( $z$ direction) were provided by a home-built Titanium:sapphire laser amplifier system with a center wavelength of $790 \mathrm{~nm}$, a pulse duration of $25 \mathrm{fs}$, and peak intensities in the range of $10^{14}$ to $10^{15} \mathrm{~W} / \mathrm{cm}^{2}$. A weak homogeneous dc field of a few $\mathrm{V} / \mathrm{cm}$ is applied along the $z$-direction. This de field not only accelerates electrons and ions to the electron and ion detectors (445 and $57 \mathrm{~mm}$ away from the interaction point, respectively) but also induces field ionization of high-lying Rydberg states populated during the strong-field interaction [12]. Additionally, a homogeneous magnetic field of 12.3 gauss ensures $4 \pi$ detection of electrons from strong-field interaction. More details on the experimental setup can be found in our previous publications $[12,25,26]$.

Previously, we developed a method to characterize Rydberg states in atoms and molecules formed during strongfield interaction. This method employs coincidence detection of Rydberg electrons released by tunnel ionization in the spectrometer dc field and single photon ionization by blackbody radiation (BBR) [12,13]. The detection of frustrated double ionization of an argon atom is depicted in Fig. 1(a). During the flight of the excited ion $\left(\mathrm{Ar}^{+*}\right)$ to the detector, the spectrometer field or BBR further releases the Rydberg electron $(e 2)$. We recorded the time-of-flight (TOF) and position information of all three particles ( $\mathrm{Ar}^{2+}$ and two electrons) with two multihit delay-line anode detectors. As shown in Fig. 1(b), the correlated signals of Rydberg electrons with their parent ions $\left(\mathrm{Ar}^{2+}\right)$ represent a long parabolic curve in the photoelectronphotoion coincidence (PEPICO) distribution. The PEPICO distribution also contains correlated signals of Rydberg 

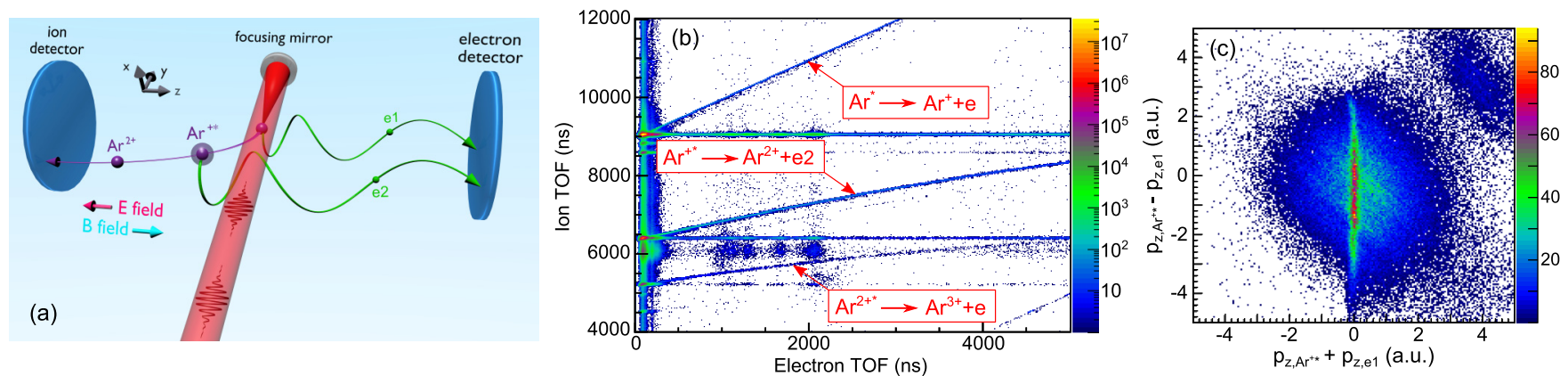

FIG. 1. (a) Schematic view of the experimental setup and the three-body detection of the dc field or BBR ionization of Ar ${ }^{+*}$ from strong laser field interaction. (b) Measured photo-electron-photo-ion coincidence distribution shows the relation between the detected electrons and ions. (c) Correlation between the retrieved momentum of $\mathrm{Ar}^{+*}$ and electrons released by strong laser fields along the laser polarization direction. Laser peak intensity is $5 \times 10^{14} \mathrm{~W} / \mathrm{cm}^{2}$, and the dc field strength is $5.8 \mathrm{~V} / \mathrm{cm}$.

electrons with $\mathrm{Ar}^{+}$and $\mathrm{Ar}^{3+}$ originating from electron trapping after strong-field single and triple ionization, as indicated in Fig. 1(b). We note that Rydberg electrons correlated with dications were also observed in separate measurements with neon atoms and acetylene molecules.

With the measured TOF and position data on the detection of Rydberg electrons and $\mathrm{Ar}^{2+}$, we retrieved the emission time $(T)$ of the Rydberg electron and the momentum vector of the $\mathrm{Ar}^{+*}$ produced during the strong-field interaction. With the assumption of Rydberg electrons ( $e 2)$ gaining negligible energy during the dc and BBR ionization, we calculate the emission time $T$ of the Rydberg electron from the acceleration function of the $\mathrm{Ar}^{+*}$ in the dc spectrometer field which yields the relation $t_{e 2}=T+\sqrt{T^{2} m_{e} / m_{\mathrm{Ar}}+t_{e 0}^{2}}$, where $t_{e 2}$ is the TOF of the Rydberg electron, and $m_{e}$ and $m_{A r}$ are the electron mass and the mass of an argon atom, respectively, $t_{e 0}=$ $\sqrt{\frac{2 m_{e} L_{e}}{E_{\mathrm{dc}} q}}$ is the TOF of a zero-momentum electron released during strong-field ionization determined by the spectrometer electric field $E_{\mathrm{dc}}, L_{e}$ is the distance from the laser focus to the electron detector, and $q$ denotes the electron charge. From the above-mentioned relations we derive the emission time $T=t_{e 2}-\sqrt{t_{e 0}^{2}+\left(t_{e 2}^{2}-t_{e 0}^{2}\right) m_{e} / m_{\mathrm{Ar}}}$ as a function of the measured $t_{e 2}$. With the retrieved survival time $T$, we can calculate the momentum of the $\mathrm{Ar}^{+*}$ gained in the laser field along the laser polarization direction, which yields $p_{z, \mathrm{Ar}^{+*}}=$ $E_{\mathrm{dc}}\left(0.5 t_{r 0}^{2}-0.5 T^{2}+t_{r} T-t_{r}^{2}\right) / t_{r}$, where $t_{r 0}$ and $t_{r}$ are TOFs of $\mathrm{Ar}^{+}$with zero initial momentum and $\mathrm{Ar}^{2+}$ from ionization of $\mathrm{Ar}^{+*}$, respectively. Because the signal of the frustrated double ionization with a small emission time $T$ overlaps with that of the strong-field ionization, we selected only the signal of frustrated double ionization with $T>150 \mathrm{~ns}$.

With the retrieved momentum of $\mathrm{Ar}^{+*}$ [from the measured data of $\mathrm{Ar}^{2+}$ and the Rydberg electron $\left.(e 2)\right]$, we can check the quality of the three-body coincidence detection of $\mathrm{Ar}^{2+}$ with two electrons for frustrated double ionization process. In Fig. 1(c) we show the momentum correlation between the $\mathrm{Ar}^{+*}$ and the electron $(e 1)$ released during the strong-field interaction with the sum and difference of their momenta. Due to momentum conservation, the momentum sum of the strong-field electron $(e 1)$ and $\mathrm{Ar}^{+*}$ from the same atom is close to zero with a narrow momentum distribution determined by the initial momentum of the argon atom.
The correlation in Fig. 1(c) shows that we achieved a high confidence in the three-body coincidence detection of two electrons and their parent ion $\mathrm{Ar}^{2+}$. For further data analysis, we applied the coincidence selection condition of $\left|p_{z, \mathrm{Ar}^{+*}}+p_{z, e 1}\right|<0.1$ a.u. for frustrated double ionization to minimize the false coincidence rate.

\section{RESULTS AND DISCUSSIONS}

\section{A. Electron trapping probability}

Figure 2(a) presents measured signals of Rydberg electrons from $\mathrm{Ar}^{*}$ and $\mathrm{Ar}^{+*}$ over emission time for a laser peak intensity of $7.9 \times 10^{14} \mathrm{~W} / \mathrm{cm}^{2}$ and a dc field strength of $3 \mathrm{~V} / \mathrm{cm}$. As reported in our recent publication, the yield of Rydberg electrons from $\mathrm{Ar}^{*}$ contains two contributions over the electron emission time: fast decay signals due to ionization in the dc spectrometer field of Rydberg states near $n_{\mathrm{dc}}=121$ and slow delay signals due to BBR-induced photoionization of Rydberg states with $n_{\mathrm{BBR}} \sim 10$ up to $n_{\mathrm{dc}}$ [12]. The signal from $\mathrm{Ar}^{+*}$ has a similar behavior but with different decay rate due to the involvement of different energy levels. Dc field ionization contributes dominantly to the signal with small emission times and causes a fast decay with an ionization rate of about $10^{-2} \mathrm{~ns}^{-1}$, while BBR-induced photoionization is responsible for the slowly decaying signal with an ionization rate of about $5 \times 10^{-4} \mathrm{~ns}^{-1}$. One clear observation is that the Rydberg electron signal from $\mathrm{Ar}^{+*}$ is much stronger than that from $\mathrm{Ar}^{*}$, even though in measurement the strong-field single ionization yield $\left(\mathrm{Ar}^{+}\right)$is about four times higher than that of the strong-field double ionization $\left(\mathrm{Ar}^{2+}\right)$.

Since the laser intensity plays an crucial role in the strongfield ionization, we performed measurements with laser peak intensities from $2 \times 10^{14} \mathrm{~W} / \mathrm{cm}^{2}$ to $7.9 \times 10^{14} \mathrm{~W} / \mathrm{cm}^{2}$. Since the ionization rate due to $\mathrm{BBR}$ is a constant, we can obtain the overall BBR ionization signal through an exponential fitting function of the BBR ionization signal over the emission time. With the obtained overall BBR ionization signal, we compare the electron trapping probability with respect to the strongfield single and double ionization processes, with normalization to the signals of strong-field single and double ionization, respectively. This ratio as a function of laser peak intensity is shown in Fig. 2(b). The electron trapping probability after 

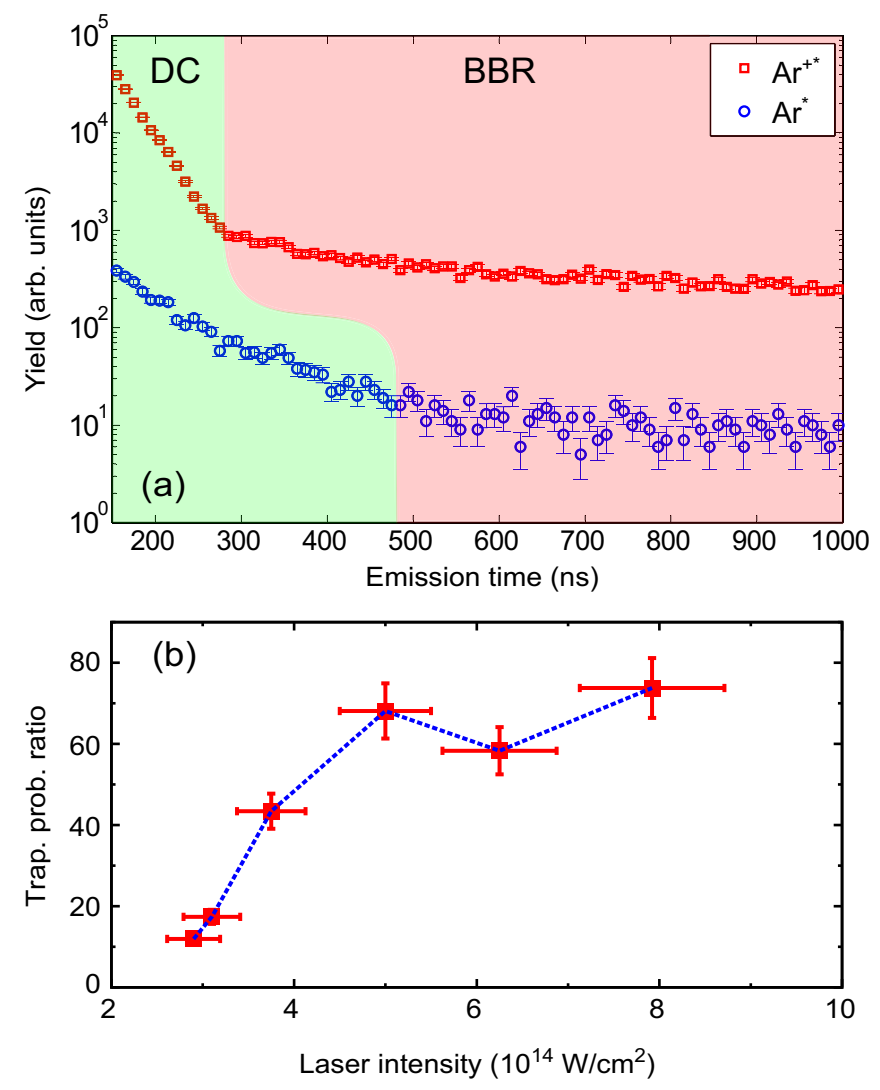

FIG. 2. (a) Distributions of measured emission time of Rydberg electrons from $\mathrm{Ar}^{*}$ (blue circles) and $\mathrm{Ar}^{+*}$ (red squares) for laser peak intensity of $7.9 \times 10^{14} \mathrm{~W} / \mathrm{cm}^{2}$ and the dc field strength of $3 \mathrm{~V} / \mathrm{cm}$. (b) The ratios of trapping probability between the single and double ionization processes as a function of laser peak intensity from measurements.

double ionization is more than one order of magnitude higher than that after single ionization. The trapping probability ratio increases with the laser intensity in the range from $2 \times 10^{14}$ to $7.9 \times 10^{14} \mathrm{~W} / \mathrm{cm}^{2}$ and saturates at about $5 \times 10^{14} \mathrm{~W} / \mathrm{cm}^{2}$.

One reason for the higher trapping probability in double ionization is obviously that two electrons can contribute to the trapping probability. Another intuitive reason for the higher trapping probability for double ionization is the different Coulomb potentials of $-1 / r$ and $-2 / r$ for the electron trapping process during single and double ionization, respectively. The Coulomb potential influences the trapping probability in two ways. First, the potential $-2 / r$ is deeper than $-1 / r$, which leads to a larger trapping volume. The spatial volume of the potential $-2 / r$ for trapping electrons with a certain near-zero kinetic energy is 8 times as that of the potential $-1 / r$. The second effect is the Coulomb focusing effect [27], which is also stronger for the potential $-2 / r$ than $-1 / r$. The Coulomb volume and focusing effects together with the doubled trapping probability lead to the strongly increased electron trapping probability after double ionization as compared to after single ionization. However, the influence of the Coulomb potential shifts the mean momenta of electrons away from the origin which will dramatically reduce the trapping probability. The shifting effect is stronger in the case of $-2 / r$ than that of $-1 / r$, which reduces the relative trapping probability. Earlier experiments and simulations have shown the multielectron influence and the breakdown of the independent electron approximation in the sequential double ionization $[28,29]$. However, a theoretical treatment of an electron in high-lying Rydberg states (with $n>100$ ) requires an extremely large simulation box size due to the scaling of the wave packet size with $n^{2}$. Therefore, it is not trivial to perform numerical simulations that mimic the situation of electron trapping to high-lying Rydberg states in a multielectron system during and after strong-field double ionization. Further experiments and $a b$ initio simulations are expected to shed light on the interpretation of the electron trapping probability ratio between single and double ionization and its dependence on the laser intensity.

\section{B. Electron momentum distributions}

Since the electron's final momentum is determined by the vector potential of the laser field at the electron's release time, measured electron momentum distributions contain temporal information of the strong-field interaction. We present electron momentum distributions along the laser polarization direction from strong-field double ionization and frustrated double ionization for three different laser peak intensities $\left(3.1,3.8\right.$, and $\left.7.9 \times 10^{14} \mathrm{~W} / \mathrm{cm}^{2}\right)$ in Figs. 3(a) and 3(b), respectively. The sharp peaks in the momentum distributions are ATI-like structures caused by the interference of electron wave packets released at different times [26,30,31]. Figure 3(a) shows that the electron momentum distribution of strong-field double ionization gradually changes from a broad double-hump structure at $3.1 \times 10^{14} \mathrm{~W} / \mathrm{cm}^{2}$ to a narrow single-hump distribution at $7.9 \times 10^{14} \mathrm{~W} / \mathrm{cm}^{2}$ as the laser intensity increases. This behavior is a manifestation of the change of the double ionization mechanism from the nonsequential to the sequential regime for multicycle laser pulses [21,26,32-34]. When the laser intensity is low, the sequential double ionization rate is low and the recollision induced double ionization dominates [21]. Sequential double ionization becomes the dominant mechanism when the laser intensity becomes strong enough [22].

For a quantitative comparison of the measured momentum distributions in Figs. 3(a) and 3(b) we retrieved their full widths at the half maximum, which are plotted as a function of laser peak intensity in Fig. 3(c) together with the electron momentum widths of strong-field single ionization. The widths in the regime of strong-field double ionization decrease monotonically with the laser intensity [green squares in Fig. 3(c)]. Such behavior has been reported previously and is due to the transition from nonsequential double ionization to sequential double ionization as the laser intensity increases [35]. However, the momentum widths of strong-field single ionization [filled red circles in Fig. 3(c)] show an opposite dependence on the laser intensity.

In the low laser intensity region of about $3 \times 10^{14} \mathrm{~W} / \mathrm{cm}^{2}$, strong-field double ionization of argon happens dominantly nonsequentially: The second electron is kicked out by the recollision of the earlier released electron [1]. In nonsequential double ionization the momentum of the two indistinguishable electrons shows strong correlation which manifests 

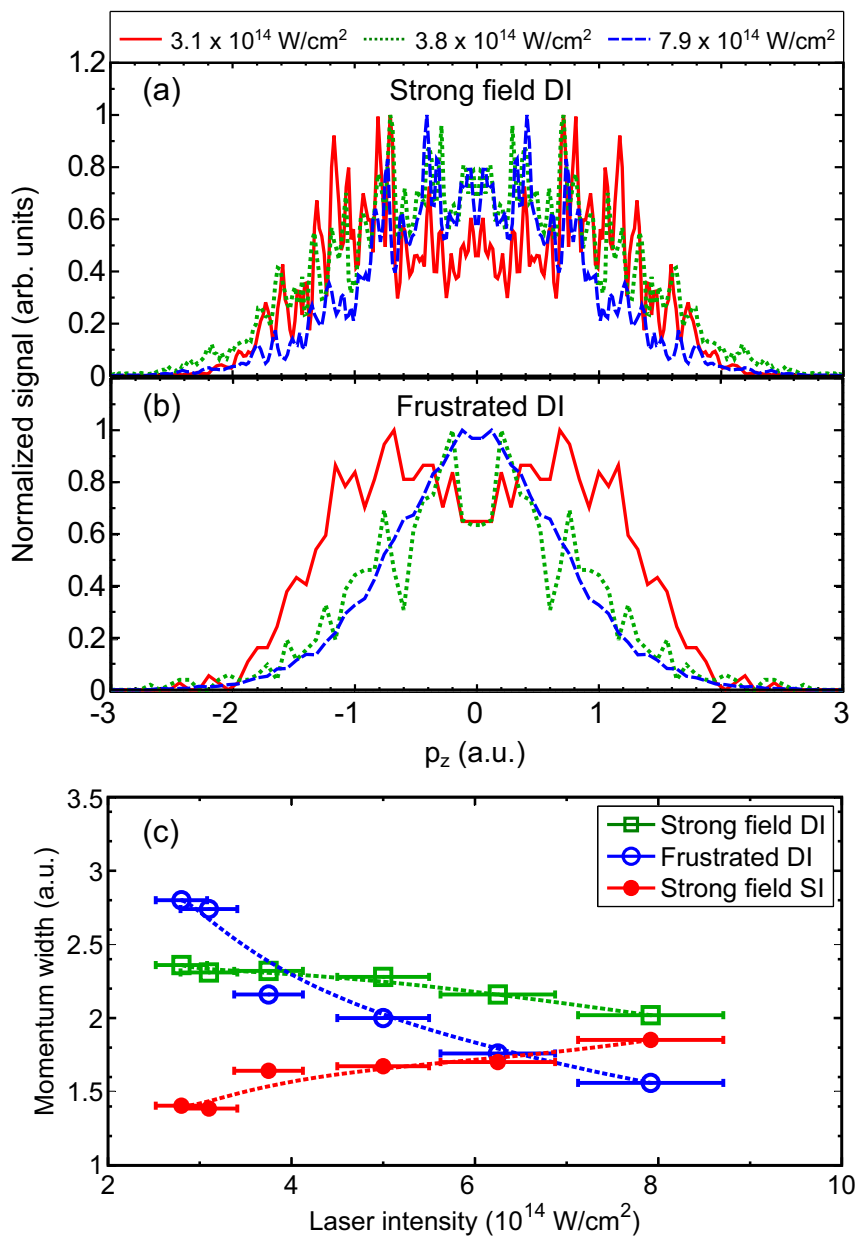

FIG. 3. Measured electron momentum distributions along the laser polarization direction from strong-field double ionization (a) and frustrated double ionization (b) for laser peak intensities of $3.1,3.8$, and $7.9 \times 10^{14} \mathrm{~W} / \mathrm{cm}^{2}$. Note that distributions with $p_{z}>0.1$ a.u. are mirrored from the negative parts, due to a technical node structure in the measured distribution in the positive momentum. (c) The width of electron momentum distribution as a function of laser peak intensity for strong-field single ionization (filled red circles), strong-field double ionization (green squares), and frustrated double ionization (open blue circles). The dashed lines (c) are fitting curves.

as a double-peak structure in the momentum distribution with peaks away from zero momentum [36,37]. In frustrated double ionization, one of the two electrons is trapped after the conclusion of the laser pulse. The electron momentum distribution with peaks away from zero leads to the suppression of the electron trapping probability because only electrons with near-zero kinetic energy can be trapped by their parent ions.

In the high laser intensity region of $7.9 \times 10^{14} \mathrm{~W} / \mathrm{cm}^{2}$, strong-field double ionization is dominated by a sequential emission dynamics: the first electron is released at the leading edge of the laser pulse and the second ionization occurs preferentially around the peak of the laser pulse [38]. Because in the measurement we cannot distinguish between the first and second electrons in strong-field double ionization due to their overlapping TOF-distributions, the measured elec- tron momentum distribution contains contributions from both electrons. Thus, the measured electron momentum width of strong-field double ionization would be broader than that of the first electron (single ionization).

Turning to the momentum widths of the first electron $(e 1)$ in the frustrated double ionization process [open blue circles in Fig. 3(c)], we observed that the momentum widths decrease with increasing laser intensity. In the low intensity region, the electron momentum width of the frustrated double ionization is larger than that of both single and double ionization. In this laser intensity region, double ionization happens nonsequentially. In nonsequential double ionization, the two released electrons share the remaining energy from the recollision. Since the trapped electron has near-zero kinetic energy, the main portion of the energy is carried by the electron $(e 1)$, which yields a broad momentum distribution of this electron. However, in the high intensity region, the momentum widths of electrons associated with the frustrated double ionization process are smaller than those associated with strong-field single and double ionization. In this laser intensity region, double ionization happens mainly sequentially, such that the two electrons are released independently. This experimental observation is a strong evidence that in the sequential double ionization regime mainly the second electron becomes trapped and the measured momentum width is mainly contributed by the first ionization step leading to a narrower electron momentum distribution, which is consistent with experimental results for molecules [17,39].

The transition from the nonsequential to the sequential double ionization scenario also explains the dependence of the trapping probability ratio on the laser intensity, shown in Fig. 2(b). In the low intensity region, the trapping probability in double ionization is less enhanced due to electrons with pronounced momentum offset induced by recollision. With increasing laser intensity more double ionization events take place sequentially, which leads to the experimentally observed increase of the electron trapping probability after double ionization.

\section{CONCLUSION}

In conclusion, we studied electron trapping processes during strong-field double ionization of argon atoms using three-body coincidence measurements. We observed a strong enhancement of the trapping probability after strong-field double ionization in comparison with single ionization. The measured intensity dependence of this enhancement indicates that in the sequential double ionization regime the trapping process is dominated by the second detached electron. In the nonsequential double ionization regime we find that the trapping probability is strongly suppressed as compared to that in the sequential double ionization regime. We attribute this to the strong correlation between the two electrons which results in momentum distributions offset from zero. To completely understand the enhancement of the trapping probability in strong double ionization, further experiments and simulations which consider multielectron effects would be beneficial.

The here applied method of coincidence detection of Rydberg states can also be used for studying electron trapping 
processes in triple or higher-order ionization processes, as well as in electron impact excitation processes [40]. Moreover, this method can be directly applied to experiments on electron trapping in laser-induced molecular ionization and dissociation processes.

\section{ACKNOWLEDGMENTS}

We thank Chii-Dong Lin, Christoph Lemell and Joachim Burgdörfer for fruitful discussions. This work was financed by the Austrian Science Fund (FWF) under Grants No. P25615N27, No. P30465-N27, and No. P28475-N27.
[1] D. N. Fittinghoff, P. R. Bolton, B. Chang, and K. C. Kulander, Phys. Rev. Lett. 69, 2642 (1992).

[2] F. Krausz and M. Ivanov, Rev. Mod. Phys. 81, 163 (2009).

[3] R. R. Jones, D. You, and P. H. Bucksbaum, Phys. Rev. Lett. 70, 1236 (1993).

[4] R. R. Jones, D. W. Schumacher, and P. H. Bucksbaum, Phys. Rev. A 47, R49 (1993).

[5] T. Nubbemeyer, K. Gorling, A. Saenz, U. Eichmann, and W. Sandner, Phys. Rev. Lett. 101, 233001 (2008).

[6] B. Manschwetus, T. Nubbemeyer, K. Gorling, G. Steinmeyer, U. Eichmann, H. Rottke, and W. Sandner, Phys. Rev. Lett. 102, 113002 (2009).

[7] B. Ulrich, A. Vredenborg, A. Malakzadeh, M. Meckel, K. Cole, M. Smolarski, Z. Chang, T. Jahnke, and R. Dörner, Phys. Rev. A 82, 013412 (2010).

[8] J. Wu, A. Vredenborg, B. Ulrich, L. P. H. Schmidt, M. Meckel, S. Voss, H. Sann, H. Kim, T. Jahnke, and R. Dörner, Phys. Rev. Lett. 107, 043003 (2011).

[9] H. Liu, Y. Liu, L. Fu, G. Xin, D. Ye, J. Liu, X. T. He, Y. Yang, X. Liu, Y. Deng, C. Wu, and Q. Gong, Phys. Rev. Lett. 109, 093001 (2012).

[10] B. Wolter, C. Lemell, M. Baudisch, M. G. Pullen, X.-M. Tong, M. Hemmer, A. Senftleben, C. D. Schröter, J. Ullrich, R. Moshammer, J. Biegert, and J. Burgdörfer, Phys. Rev. A 90, 063424 (2014).

[11] Q. Li, X.-M. Tong, T. Morishita, C. Jin, H. Wei, and C. D. Lin, J. Phys. B: Atom. Mol. Opt. Phys. 47, 204019 (2014)

[12] S. Larimian, S. Erattupuzha, C. Lemell, S. Yoshida, S. Nagele, R. Maurer, A. Baltuška, J. Burgdörfer, M. Kitzler, and X. Xie, Phys. Rev. A 94, 033401 (2016).

[13] S. Larimian, C. Lemell, V. Stummer, J.-W. Geng, S. Roither, D. Kartashov, L. Zhang, M.-X. Wang, Q. Gong, L.-Y. Peng, S. Yoshida, J. Burgdörfer, A. Baltuška, M. Kitzler, and X. Xie, Phys. Rev. A 96, 021403(R) (2017).

[14] B. Manschwetus, H. Rottke, G. Steinmeyer, L. Foucar, A. Czasch, H. Schmidt-Böcking, and W. Sandner, Phys. Rev. A 82, 013413 (2010).

[15] A. Emmanouilidou, C. Lazarou, A. Staudte, and U. Eichmann, Phys. Rev. A 85, 011402(R) (2012).

[16] Q. Cheng, X. Xie, Z. Yuan, X. Zhong, Y. Liu, Q. Gong, and C. Wu, J. Phys. Chem. A 121, 3891 (2017).

[17] W. Zhang, Z. Yu, X. Gong, J. Wang, P. Lu, H. Li, Q. Song, Q. Ji, K. Lin, J. Ma, H. Li, F. Sun, J. Qiang, H. Zeng, F. He, and J. Wu, Phys. Rev. Lett. 119, 253202 (2017).

[18] K. N. Shomsky, Z. S. Smith, and S. L. Haan, Phys. Rev. A 79, 061402(R) (2009).

[19] A. Chen, H. Price, A. Staudte, and A. Emmanouilidou, Phys. Rev. A 94, 043408 (2016).

[20] S. Larochelle, A. Talebpour, and S. L. Chin, J. Phys. B: Atom. Mol. Opt. Phys. 31, 1201 (1998).
[21] T. Weber, M. Weckenbrock, A. Staudte, L. Spielberger, O. Jagutzki, V. Mergel, F. Afaneh, G. Urbasch, M. Vollmer, H. Giessen, and R. Dörner, J. Phys. B: Atom. Mol. Opt. Phys. 33, L127 (2000).

[22] W. Becker, X.-J. Liu, P. J. Ho, and J. H. Eberly, Rev. Mod. Phys. 84, 1011 (2012).

[23] R. Dörner, V. Mergel, O. Jagutzki, J. U. L. Spielberger, R. Moshammer, and H. Schmidt-Böcking, Phys. Rep. 330, 95 (2000).

[24] J. Ullrich, R. Moshammer, A. Dorn, R. Doerner, L. P. H. Schmidt, and H. Schmidt-Boecking, Rep. Prog. Phys. 66, 1463 (2003).

[25] X. Xie, K. Doblhoff-Dier, S. Roither, M. S. Schöffler, D. Kartashov, H. Xu, T. Rathje, G. G. Paulus, A. Baltuška, S. Gräfe, and M. Kitzler, Phys. Rev. Lett. 109, 243001 (2012).

[26] X. Xie, S. Roither, D. Kartashov, E. Persson, D. G. Arbó, L. Zhang, S. Gräfe, M. S. Schöffler, J. Burgdörfer, A. Baltuška, and M. Kitzler, Phys. Rev. Lett. 108, 193004 (2012).

[27] T. Brabec, M. Y. Ivanov, and P. B. Corkum, Phys. Rev. A 54 2551(R) (1996); D. Comtois, D. Zeidler, H. Pépin, J. Kieffer, D. Villeneuve, and P. Corkum, J. Phys. B: Atom. Mol. Opt. Phys. 38, 1923 (2005).

[28] A. N. Pfeiffer, C. Cirelli, M. Smolarski, X. Wang, J. H Eberly, R. Dörner, and U. Keller, New J. Phys. 13, 093008 (2011).

[29] Y. Zhou, C. Huang, and P. Lu, Opt. Express 20, 20201 (2012).

[30] J. S. Parker, B. J. S. Doherty, K. T. Taylor, K. D. Schultz, C. I. Blaga, and L. F. DiMauro, Phys. Rev. Lett. 96, 133001 (2006).

[31] D. G. Arbó, K. L. Ishikawa, K. Schiessl, E. Persson, and J. Burgdörfer, Phys. Rev. A 82, 043426 (2010).

[32] M. Lein, E. K. U. Gross, and V. Engel, Phys. Rev. Lett. 85, 4707 (2000).

[33] K. Zrost, A. Rudenko, T. Ergler, B. Feuerstein, V. L. B. de Jesus, C. D. Schröter, R. Moshammer, and J. Ullrich, J. Phys. B: Atom. Mol. Opt. Phys. 39, S371 (2006).

[34] M. Kübel, K. Betsch, N. G. Kling, A. Alnaser, J. Schmidt, U. Kleineberg, Y. Deng, I. Ben-Itzhak, G. Paulus, T. Pfeifer et al., New J. Phys. 16, 033008 (2014).

[35] A. Rudenko, T. Ergler, K. Zrost, B. Feuerstein, V. L. B. de Jesus, C. D. Schröter, R. Moshammer, and J. Ullrich, J. Phys. B: Atom. Mol. Opt. Phys. 41, 081006 (2008).

[36] A. Rudenko, V. L. B. de Jesus, T. Ergler, K. Zrost, B. Feuerstein, C. D. Schröter, R. Moshammer, and J. Ullrich, Phys. Rev. Lett. 99, 263003 (2007)

[37] A. Staudte, C. Ruiz, M. Schöffler, S. Schössler, D. Zeidler, T. Weber, M. Meckel, D. M. Villeneuve, P. B. Corkum, 
A. Becker, and R. Dörner, Phys. Rev. Lett. 99, 263002 (2007).

[38] M. S. Schöffler, X. Xie, P. Wustelt, M. Möller, S. Roither, D. Kartashov, A. M. Sayler, A. Baltuska, G. G. Paulus, and M. Kitzler, Phys. Rev. A 93, 063421 (2016).

[39] H. Hu, S. Larimian, S. Erattupuzha, J. Wen, A. Baltuška, M. Kitzler-Zeiler, and X. Xie, Phys. Rev. Research 1, 033152 (2019).
[40] B. L. Moiseiwitsch and S. J. Smith, Rev. Mod. Phys. 40, 238 (1968).

Correction: The two values for distances away from the interaction point in Sec. II were incorrectly given and have been set right. The caption to Fig. 2 should end after the word "measurement." The label for electron 1 has been set consistently. The last sentence of text contained an error and has been fixed. 